\title{
FOUR CASES OF CONGENITAL DEFORMITY OF THE HEAR'T DUE TO ANOMALOUS MECHANICAL INFLUENCES IN THE MALFORMED FCETUS. ${ }^{1}$
}

\author{
By J ANE I. Robertson, M.B., Carnegie Research Scholar, \\ Western Asylum's Research Institute, Glasgow.
}

\section{(Plate XV.)}

THE following is an account of four fotal hearts from three cases of anencephaly and one of ectopia viscerum all about the seventh month of development. The general appearance and arrangement of all four specimens deviate distinctly from the normal, but are essentially similar to one another, their differences being merely matters of degree. The fourth case, however, shows some additional peculiarities which must probably be correlated with the condition of this foetus not present in the three others, namely, the marked ventral and dorsal ectopion. The three cases of anencephaly will be discussed first, the fourth specimen being dealt with more easily later. ${ }^{2}$

Plate XV. Fig. 1 gives an excellent representation from a ventral view of the general appearance of these hearts as they lie in their thoracic cavities. On first glancing at these specimens one has the impression that instead of looking at the ventral, one is examining the right lateral, surface of the organ, and instinctively an attempt is made to rectify the apparent malposition by rotating the heart from left to right through about one right angle. This rotation at once approximately corrects the topographical relations of the various parts of the heart to one another and to the surrounding structures, but the organ will not maintain this position of itself and did not occupy it in the foetus, and, when so corrected, presents a comparatively sharp keellike ventral surface (Fig. $1(d)$ ). To express it as concisely as possible, these three specimens present externally no abnormalities beyond those involved by their right and left surfaces having become ventral and dorsal respectively, and their ventral and dorsal surfaces, ipso facto

\footnotetext{
1 Received July 24, 1913.

2 I have to acknowledge my indebtedness to Dr. Anderson, pathologist to the Victoria Infirmary, Glasgow, for the hearts of two of the anencephalic cases and for that from the case of ectopia viscerum.
} 
left and right respectively (Plate XV. Fig. 1). The arrangement of the great veins and great arterial trunks are normal, except that the origin of the pulnonary artery is placed more to the left of that of the aorta than is usual (compare Fig. $1(d)$ and $(h)$ ), and that there is a

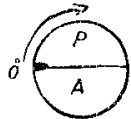

(a)

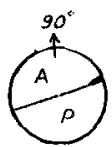

(b)

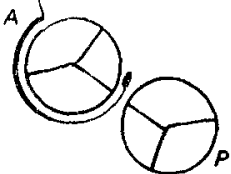

(c)

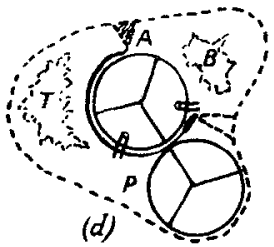

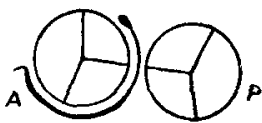

(g)

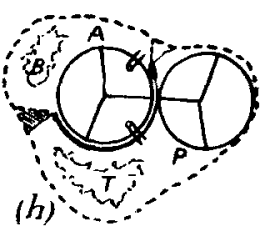

(e)

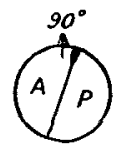

(f)

Fit. 1.-Diagram (a) shows the normal relations of the pulmonary artery (P) and the aorta (A) at the distal extremity of the bulbus cordis, and diagram (b) the relations of the same vessels at its proximal extremity : the rotation of the two vessels round one another between these two levels being indicated by the position of the rounded knob on the aortico-pulmomary septum. Diagram (c) shows the normal relations of the great vessels at their proximal extremities, the insertion of the aortico-pulmonary septum, and of the ventral part of the interventricular septum being indicated by the rounded knob, while the spindle-shaped thickening represents the pars membranacea septi; diagram $(d)$ is an amplification of diagram $(c)$ showing the relations of the apertures and chambers at the base of the heart.

Diagram $(o)$ shows the relations of the pulmonary artery $(P)$ and aorta $(A)$ at their distal extremities, and diagram $(f)$ the relations of the same vessels at their proximal extremities, in the four cases of anencephaly: the rotation of the two vessels rond one another between these two points is indicated by the position of the rounded knoh on the aortico-pulmonary septum. The decreased rotation in the anencephalic cases as compared with diagrams $(a)$ and $(b)$ of the normal series will be perceived at once. Diagram $(g)$ shows the positions of the great vessels proximally, the insertion of the aortico-pulmonary septum, and of the ventral part of the interventricular septum beings indicated by the rounded knob, while the spindle-shaped thickening represents the pars membranaee septi ; diagram $(h)$ is an amplification of diagram $(g)$ showing the relations of the apertures and chambers at the bases of the four hearts from cases of anencephaly.

The positions of the coroury arteries are indicated in diagrams $(d)$ and $(h)$.

$A$, aorta ; $B$, bicuspid opening ; $P$, pulmonary artery ; $Z$, tricuspid opening .

marked straightening of the two arterial trunks and of the ductus arteriosus, giving them an appearance of unusual length. Plate XV. Fig. 1 shows the great vessels cut across just below the point where the ductus arteriosus and the aorta become continuous. In order to show the position of the interventricular sulcus on the left border of the beart, this specimen had to be tilted round somewhat to the right, which places the pulmonary artery apparently more ventrally, in 
relation to the aortic trunk, than it really is. The immediate deduction to be drawn, therefore, from the comparative success attending the attempted reposition of these hearts and from the absence of any obvious malformations, is that the main errors of development to be dealt with here are, (1) an increase-amounting approximately to one right angle - of the usual degree of right-to-left pivoting of the ventricular loop (compare (Fig. $1(d)$ and $(h)$ ), and (2) an unusual straightening of the great arterial trunks. Save for a large patent foramen ovale in each, these three specimens presented no abnormalities whatsoever. The relative positions of the various compartments are indicated in Fig. $1(h)$, the variation in the three cases being too slight to warrant separate diagrams.

Apparently, then, except for the actual position adopted by the cardiac loop and for a certain degree of extension of the great vessels, the development of these hearts themselves as a whole must be considered to have occurred along normal lines-that is to say, we have here to deal, not with any malformations of the heart itself, but with adaptations imposed upon an essentially normal organ by errors in development of more or less remote parts of the foetus. The only common factor, however, present in all these individuals that can have exerted any unusual influence on the heart, is the condition of anencephaly, with its concomitant extension of the foetal neck. It would, therefore, seem natural to correlate the two conditions and to consider the cardiac adaptations as secondary to the anencephaly. Several considerations would point to the reasonableness of this conclusion. The development of these hearts must obviously have proceeded throughout with a minimum degree of disturbance, save that due to the faulty development of the cranial end of the nervous system and axial skeleton of the fotus. This abnormal condition of the cranial end of the foetus would be accompanied by. a shortening, at least, of the normal duration of the period of marked cervical flexure, if not by its entire abeyance, and subsequently an unusual degree of extension would be applied to the great vessels at the arterial end of the cardiac loop throughout development. We know that in the course of normal development the cardiac loop as a whole undergoes a certain degree of right to left displacement (1889 ${ }^{1}$ ), if now an abnormal extension of the great vessels be supposed to exist during the occurrence of this right-to-left rotation, then presumably the extension would tend to exaggerate that movement, and so help to bring about the increasedly dorso-ventral relations of the left and right ventricles noted in these cases. Similarly, the extension of the cervical region would account for the unusually erect position of the great vessels and ductus arteriosus, and thus also for their apparent increase in length.

Another factor to be considered is the effect of this abnormal extension of the great vessels on their normal degree of rotation round

15-3i. of PATT. - Vor. xvir. 
one another from their distal to their proximal extremities. As I have indicated elsewhere $\left(1913^{2}\right)$ the rotation of the great vessels round one another from dorso-ventral (pulmonary artery and aorta respectively) at their distal, to left ventral (pulmonary artery) and right dorsal (aorta) at their proximal extremities, would seem to be brought about by the kinking and asymmetrical expansion of the primitive bulbus cordis. The object of this rotation of the great vessels is to enable the venous blood from the right side of the heart to reach the posterior (sixth) pair of aortic arches (pulmonary arteries). For this rotation of the vessels to occur, a certain degree of kinking or folding of the developing bulbus cordis is necessary to allow of the proper degree of spiral development of the aortico-pulmonary septum. If, however, an unusual degree of extension of the arterial end of the heart be present throughout development, then the normal amount of spiral formation of the aortic-pulmonary septum will be more or less inhibited, with the result that there will be a more or less decreased rotation of the great vessels round one another. This is clearly shown in Fig. 1, where diagrams $(\alpha)$ to $(c)$ represent the normal, and diagrams $(e)$ to $(g)$ the anencephalic series. Of course it must be borne in mind that both the increased right-to-left torsion of the whole cardiac loop and the diminished spiral formation in the bulbus cordis, are coincident and correlated modifications, not isolated and independent phenomena. These anencephalic cases can now be classified according to Rokitanski's (1875 ${ }^{3}$ ) arrangement of transpositions of the great vessels. Rokitanski has not actually figured any such cases as these, but they fall naturally into his series $A$, of "corrected" transpositions-that is, although the vessels are not in their normal relations to one another, yet they open out of the correct ventricles (Fig. 1).

The specimen shown in Plate XV. Figs. 2, 3, and 4 may now be discussed in the light of the above remarks. It will be seen after a little scrutiny that this specimen is essentially an instance of the same type of adaptation of a normal heart as the three preceding ones. There is the same exaggeration of the right-to-left rotation of the ventricular loop and the same straightening and apparent lengthening of the great vessels. Plate XV. Fig. 2 shows a ventral view of this specimen which has, however, been tilted somewhat to the right to show the interventricular sulcus, with the result that the pulmonary artery has the appearance of being more in front of the aorta than it really is. The only striking differences between Plate XV. Figs. 1 and 2 are the rounded bulging shape of the left ventricular margin and the extraordinarily elongated-one might almost say dislocatedappearance of the right auricle and inferior cava in the latter as compared with these structures in the former figure. If Plate XV. Figs. 2 and 3 , the latter showing the same specimen from a dorsal view, be examined, it will be found that all the various vessels and 
chambers are present and apparently all possess the normal inlet and outlet apertures; these facts will be further established if Plate XV. Fig. 4, which shows the interior of both auricular cavities, be carefully scrutinised. It will be observed that here, too, as was noted for the three preceding cases, there is a large persistent foramen ovale between the two auricles (see text for Plate). On opening and carefully examining the various chambers of this specimen, no abnormalities are noted save that the right auricle is displaced apparently towards the apex of the ventricle, the left auriculoventricular opening (Plate XV. Fig. 4, L.A.V.O.) lies to the left of and cranial to the right opening (Plate XV. Fig. 4, R.A.V.O.) and that the right ventricle is disproportionately long compared to its width, and forms the whole of the ventricular apex (Plate XV. Figs. 2 and 3, R.V.). Of course here, as in the other specimens, the right and left lateral surfaces of the heart have become ventral and dorsal respectively, but this must be regarded as an adjustment of position, not as an actual malformation of the organ. Apparently, therefore, we are here dealing with a heart essentially similar to the three already examined save for one obvious difference. This difference may be expressed forcibly if somewhat picturesquely by saying that this specimen suggests the occurrence of a landslide of the right posterior part of the heart during development. Rough though the simile may be, it states the case exactly, and moreover suggests at once the peculiarity characteristic of this foetus as compared with the other three, namely, the ventral and dorsal ectopion and the complete displacement ventrally and downwards (towards the feet) of all the abdominal viscera. Here, therefore, instead of the mechanical effect of a cervical extension on the arterial end of the heart, there has been in this case, a downward tension exerted on the venous end of the heart by a hernia of the liver and abdominal contents. The result, as is well shown in Plate XV. Figs. 2, 3, and 4, and as has already been mentioned, is a downwards (towards the feet) and somewhat ventral displacement of the right auricle as a whole and of the right auriculo-ventricular opening, while correlated with this there is a marked lengthening along the long axis of the heart, of the right ventricle which forms the apex of the organ (Plate XV. Figs. 2 and 3, R.V.) ; similarly, there has been a slight degree of displacement downwards and to the right of the right pulmonary veins (Plate XV. Fig. 4, R.P.V.). Accordingly there is here an adjustment of the arterial trunks and cardiac loop, similar to that already described for the three preceding cases, combined with an adjustment of the inferior vena cava, both being due to a condition of ectopia viscerum. In this case the tension posteriorly is exerted in a downward direction (towards the feet) and ventrally-that is downwards and to the left -as it is due largely to the ventral hernia of the liver. Here again therefore the normal right-to-left rotation of the developing heart $\left({ }^{1}\right)$ 
will be exaggerated by the action of this abnormal tension, while on the other hand the tension plus the exaggerated ventricular rotation will also tend to straighten the bulbus cordis, and thus, as we have seen, modify the usual degree of rotation of the great arterial trunks round one another. Thus, in the fourth case under discussion, we have a cardiac condition essentially comparable to that of the anencephalic cases, but due to an abnormal influence acting on the opposite end of the heart. This specimen falls into the same place in Rokitanski's scheme as the preceding ones, and Fig. 1, diagram (h), is equally applicable to it.

\section{SUMmary.}

The marked resemblance of these four hearts to one another is of great interest, as is also the conclusion arrived at that, in all four cases, we are dealing with a purely mechanical adjustment and not with a real developmental anomaly of the heart. The resemblance of these cases is due to the fact that in each instance it was a normally developing heart that was subjected to a similar abnormal mechanical influence imposed upon it, but not inherent to it as the result of any actual malformation of the organ itself. The three first specimens, as we have seen, are probably instances of adjustments of a normal heart to a mechanical strain exerted on its arterial end, while the fourth specimen is an instance of an adjustment of the normal heart to a mechanical strain exerted on its venous end. It is to be noted that probably the tension exerted by the hernia of the abdominal contents would begin approximately at the same time as that exerted by the extension of the neck. It is impossible to date the initiation of the cervical extension in cases of anencephaly-it is conceivable that it may occur extremely early--but, normally, cervical extension is considered to occur about the fourth or fifth week of embryonic life. Similarly there would hardly be much strain on the venous end of the heart till the liver had attained some size and come to protrude to some extent from the abdomen, two conditions that would hardly be fulfilled before the fifth week. Born $\left(1889^{1}\right)$ considers the various septa of the heart to be complete about the sixteenth or seventeenth day of foetal life, so that the comparatively late onset of the pathological influences would account for the relatively normal development of these four hearts.

\section{REFERENCES.}

1. Born, G. . . . . . . "Beitr. z. Entwickelungsgeschichte des Säugetierherzens," Arch. f. Mikro. Anat., Bonn, 1889, Bd. xxxiii. S. 284.

2. Robertbon, Jane I. . " "The Comparative Anatomy of the Bulbus Cordis with special Reference to Abnormal Positions of the great Vessels in the Human Heart," Journ. Path. and Bacteriol., Cambridge (1913-14), vol, xviii. p. 191. 


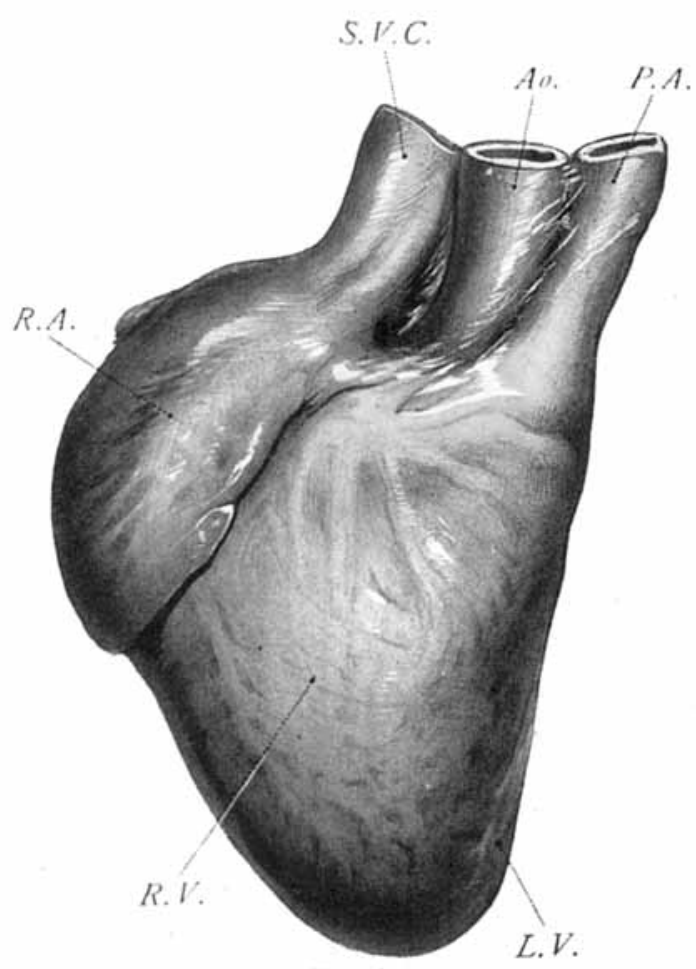

FIG. 1.

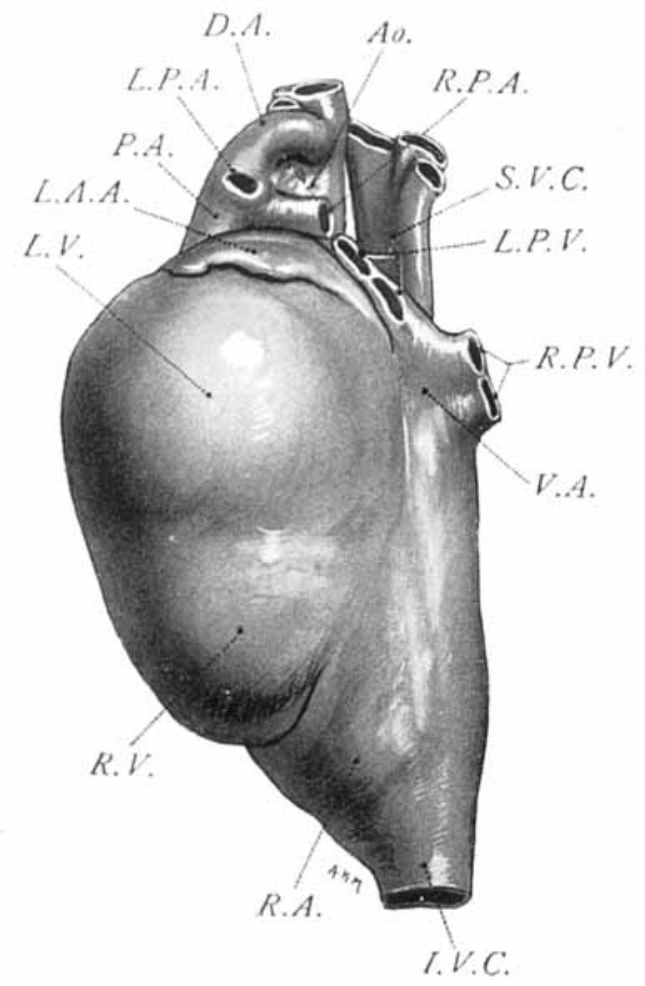

Fic. 3.

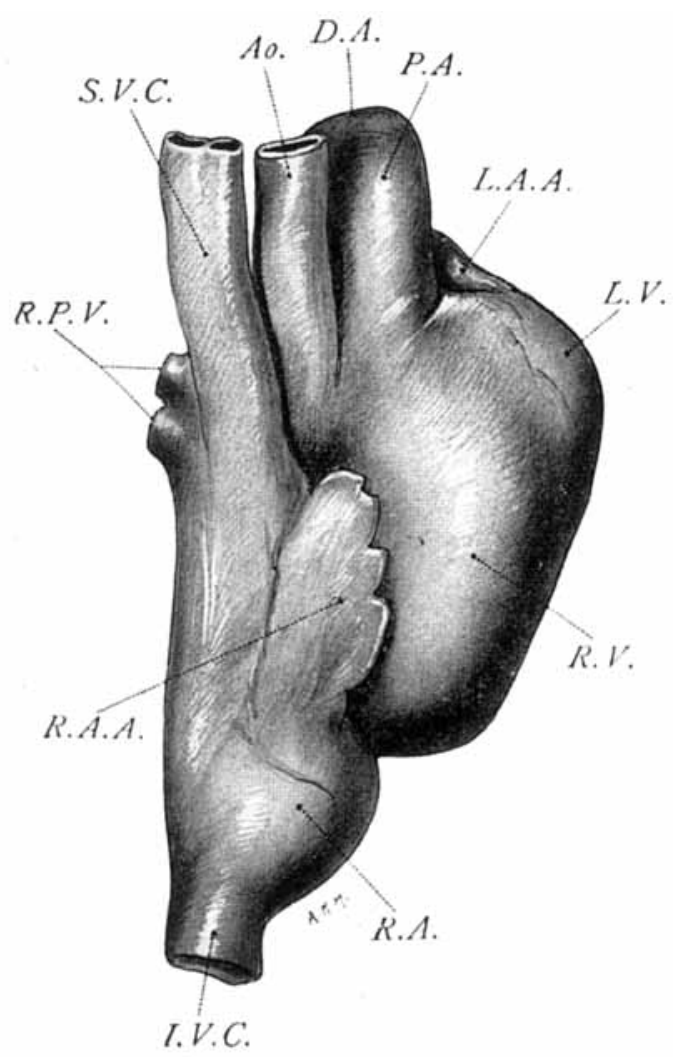

Fig. 2.
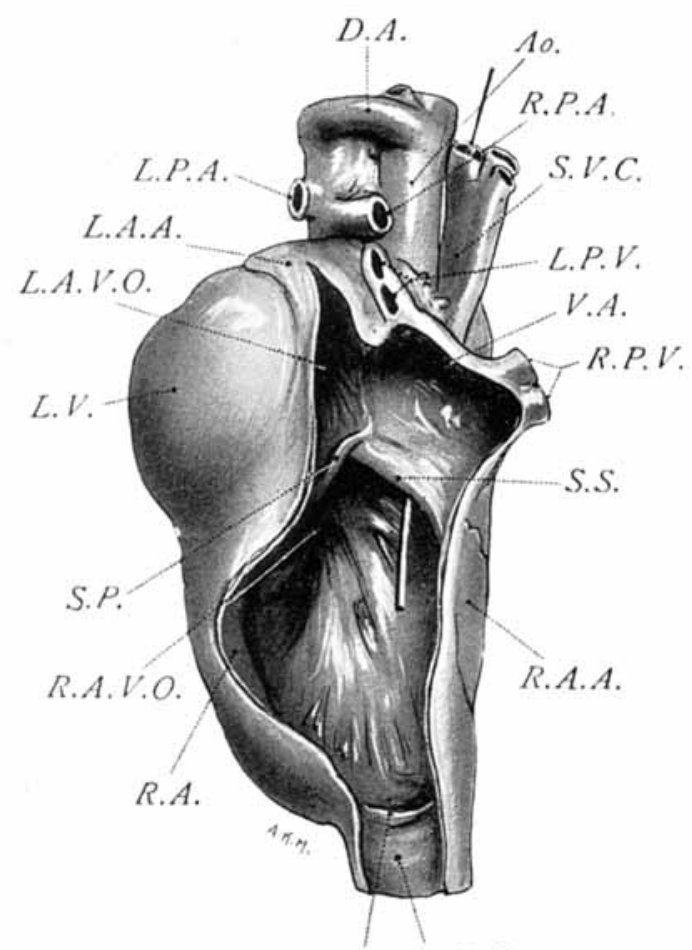

R.V.V. I. V.C.

Fig. 4. 


\section{Rokitanski, C. . . . " "Die Defecte der Scheidewaende des Herzens," Wien, 1875.}

\section{DESCRIPTION OF PLATE XV.}

\section{REFERENCE LeTTERS.}

Ao., aorta ; D.A., ductus arteriosus ; I.V.C., inferior vena cava ; L.A.A., left auricular appendix; L.A.V.O., left auriculo-ventricular orifice ; L.P.A., left pulmonary artery ; L.P.V., left pulmonary veins ; L.V., left ventricle; P.A., pulmonary artery; R.A., right auricle; R.A.A., right auricular appendix $;$ R.A.V.O., right auriculoventricular orifice; R.P.A., right pulmonary artery ; R.P.V., right pulmonary veins ; R.V., right ventricle; R.V.V., right venous valve ; S.P., septum primum; S.S., septum secundum; S.V.C., superior vena cava; V.A., vestibule of left auricle.

FiG. 1.-Drawing of the heart from a case of anencephaly, ventral view. In order to allow the interventrieular sulcus to be seen, the specimen is tilted a little to the right, which gives the pulmonary artery the appearance of being situated somewhat more in front of the aorta than it is in reality. Note the marked right-to-left displacement of the whole heart.

FIG. 2.-Drawing of the heart from a case of ectopia viscerum, ventral view. In order to allow the interventricular sulcus to be seen, this specimen is tilted a little to the right, which gives the pulmonary artery the appearance of being situated somewhat more in front of the aorta than it is in reality. Particularly to be noted in this specimen are-the marked right-to-left displacement of the whole heart; the straightened elongated appearance of the great vessels; the irregular appearance of the ventricular part of the heart; the marked elongation of the right ventricle; the posterior displacement (towards the feet) of the right auricle, right auricular appendix and inferior vena cava.

FIG. 3.-Drawing of the same specimen as Plate XV. Fig. 2, dorsal view. Note the position of the left ventricle as a sort of dorsal hump on the right ventricle, while the latter chamber is so elongated that it forms the whole of the apex of the heart. The posterior displacement (towards the feet) of the right auricle and inferior vena cava is here very evident, and so too is the degree of separation of the right and left pulmonary venous trunks.

Fig. 4.-Drawing of the same specimen as Plate XV. Figs. 2 and 3 from a somewhat right dorsal view. The inferior vena cava and right and left auricles are laid open and their cavities exposed. The displacement of the right auricle downwards (to the feet) and ventrally, till it lies posterior and ventral to the Jeft auricle is well seen. These relations of the two auricles are rendered very evident by the relative positions of the two auricular appendices and of the two auriculo-ventricular orifices. It is possible to obtain so clear a view of the interior of the right auricle owing to the presence of a large foramen ovalo whose left ventral and right dorsal margins are demareated by a rudimentary septum primum (S.P.), and septum secundum (S.S.), respectively. These septa and the foramen ovale owe their oblique position, of course, to the relative displacement of the auricles. A seeker is shown passing along the superior vena cava into the right auricle, where it is seen lying ventral to the foramen ovale. The left auriculo-ventricular orifice (L.A.V.O.) lies to the left and cranial to the right orifice $(R . A . V . O$, while the vestibular portion of the left auricle (V.A.) shows evidence of unusual elongation. At the opening of the inferior vena cava, part of the right venous valve (R.V.V.) will be observed. 